\title{
Development and Comparison of Fiber-Optic Sensors in Different Shapes for Remote Measurement of Gamma Radiation
}

\author{
Chan Hee Park, Rinah Kim, ${ }^{1}$ Han Young Joo, ${ }^{1}$ and Joo Hyun Moon ${ }^{1 *}$ \\ Korean Association for Radiation Application, 77, Seongsoo 1-ro, Seongdong-gu, Seoul 04790, Republic of Korea \\ ${ }^{1}$ Department of Nuclear \& Energy System Engineering, Dongguk University-Gyeongju, \\ 123 Dongdae-ro, Gyeongju, Gyeongbuk 38066, Republic of Korea
}

(Received March 3, 2015; accepted February 29, 2016)

Keywords: fiber-optic sensor, gamma radiation, plastic optical fiber, inorganic scintillator, sensing probes, reflector

For safety reasons, it is desirable to measure radiation as remotely as possible. For remote measurement of gamma radiation, the fiber-optic sensor (FOS) was developed in this study by combining a plastic optical fiber and an inorganic scintillator. To identify the best shape of the sensing probe of the FOS in terms of its detection efficiency, three different shapes of probe were fabricated using an inorganic scintillator: a cylinder and two bundles of different lengths (30 and 10 $\mathrm{mm})$. To identify the best reflector for the sensing probe, the side of each probe was wrapped with one of three different reflectors: Teflon tape, TYVEK 1056D, and aluminum foil. In this study, a total of twelve different sensing probes were prepared: three different shapes of sensing probes with three different reflectors, and three different shapes of the sensing probes without reflectors. To measure gamma radiation, a cesium-137 source $(1 \mu \mathrm{Ci})$ in a disk shape, $26 \mathrm{~mm}$ in diameter, was attached to the end of the sensing probes. The measurements showed that bundle shape II (a bundle of 26 inorganic scintialltors, $1 \mathrm{~mm}$ in diameter and $10 \mathrm{~mm}$ in length) was the best shape for the sensing probe, and aluminum foil was the best reflector.

\section{Introduction}

Fiber-optic sensors (FOSs) have many advantages for measuring physical quantities. Because an FOS does not include a conductor of electricity, it is not interfered with electromagnetic waves generated by surrounding electronic devices, and it is free from risks of electrical short circuits and electric shock. It is easy to fabricate and light to carry. It can measure almost every type of physical quantity. In particular, it has good sensitivity because it uses short-wavelength light to measure physical quantities. ${ }^{(1)}$ It can also make remote measurements kilometers away because the signals are minimally attenuated in the optical fiber.

The most important feature of the FOS is that it could be used under harsh conditions such as those of high electromagnetic waves, high temperature, or high humidity, where conventional electronic devices cannot be operated. ${ }^{(2-5)}$ For nuclear power plants, the FOS could be used to measure radiation in conditions of high temperature and high humidity during accidents.

"Corresponding author: e-mail: jhmoon86@dongguk.ac.kr 
For remote measurement of gamma radiation, an FOS was developed in this study by combining a plastic optical fiber and an inorganic scintillator. Three different shapes of FOS sensing probes were fabricated using an inorganic scintillator. The side of each sensing probe was wrapped with one of three different reflectors: Teflon tape, TYVEK 1056D, and aluminum foil. For measurement of gamma radiation, a total of twelve different sensing probes were prepared: three different shapes of sensing probes with three different reflectors, and three different shapes of sensing probes without reflectors. A cesium-137 source $(1 \mu \mathrm{Ci})$ in a disk shape, $26 \mathrm{~mm}$ in diameter, was attached to the end of the sensing probes. Measurements were analyzed to identify the best shape of the sensing probe and the best reflector of the FOS in terms of detection efficiency.

\section{Materials and Methods}

In this study, the three different shapes of FOS sensing probe were fabricated using an inorganic scintillator $(\mathrm{Lu}, \mathrm{Y})_{2} \mathrm{SiO}_{5}: \mathrm{Ce}$ (LYSO:Ce, Advanced Microwave Technologies Solution Co., Ltd, Korea); ${ }^{(6)}$ a cylinder $26 \mathrm{~mm}$ in diameter and $30 \mathrm{~mm}$ in length (Cylinder shape); a bundle of 26 inorganic scintillators $1 \mathrm{~mm}$ in diameter and $30 \mathrm{~mm}$ in length (Bundle shape I); and a bundle of 26 inorganic scintillators $1 \mathrm{~mm}$ in diameter and $10 \mathrm{~mm}$ in length (Bundle shape II), as shown in Fig. 1. The main characteristics of the inorganic scintillator LYSO:Ce are listed in Table 1.

The two bundle-shaped sensing probes were fabricated by putting together 26 inorganic scintillators, $1 \mathrm{~mm}$ in diameter, using epoxy resin. The tapered optical fiber (Edmund Optics) was attached to the end of each bundle-shaped sensing probe to make it easier to collect light generated in the probe. All the sensing probes were connected to a plastic optical fiber with a SMA 905 connector (Thorlabs Co.). To increase the FOS's detection efficiency, the side of each sensing probe was wrapped with one of three different reflectors: Teflon tape (DuPont Co.), TYVEK 1056D

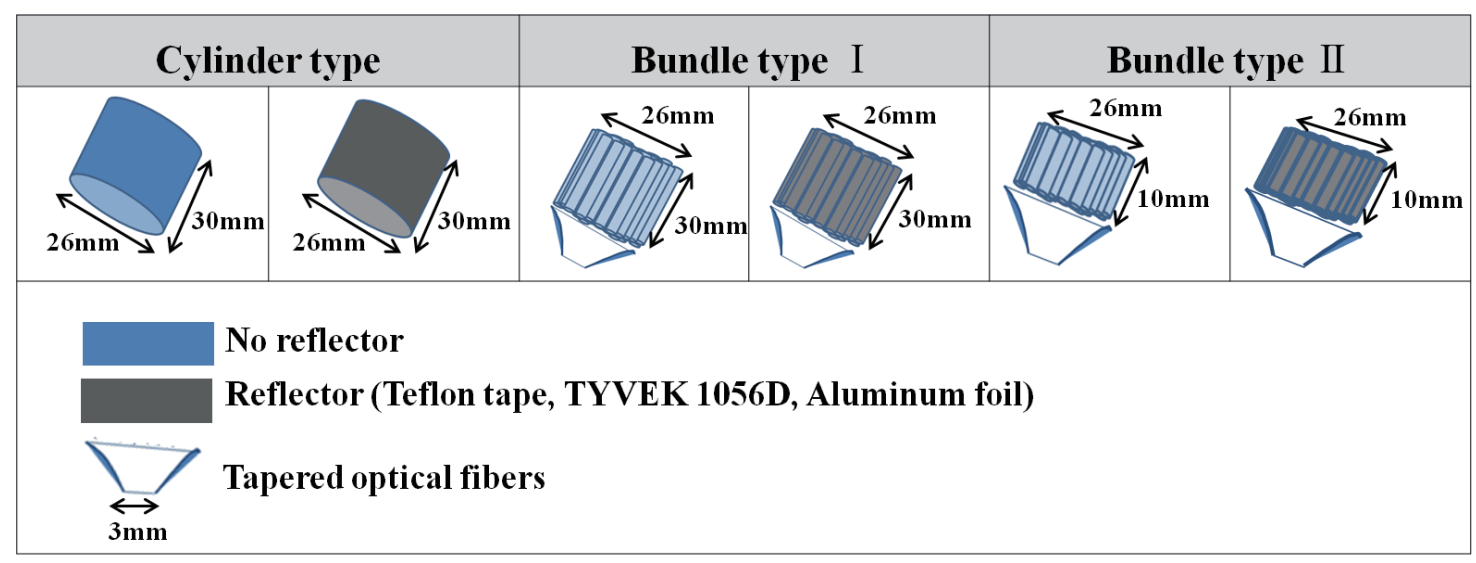

Fig. 1. (Color online) Three different shapes of FOS sensing probes.

Table 1

Main characteristics of the inorganic scintillator LYSO:Ce.

\begin{tabular}{ccccccc}
\hline $\begin{array}{c}\text { Effective } Z \\
\text { number }\end{array}$ & $\begin{array}{c}\text { Density } \\
\left(\mathrm{g} / \mathrm{cm}^{3}\right)\end{array}$ & $\begin{array}{c}\text { Melting point } \\
\left({ }^{\circ} \mathrm{C}\right)\end{array}$ & $\begin{array}{c}\text { Refractive } \\
\text { index }\end{array}$ & $\begin{array}{c}\text { Wavelength } \\
\text { peak }(\mathrm{nm})\end{array}$ & $\begin{array}{c}\text { Light yield }(\%) \\
\text { relative to NaI:T1 }\end{array}$ & $\begin{array}{c}\text { Decay time } \\
(\mathrm{ns})\end{array}$ \\
\hline 65 & 7.20 & 2100 & 1.82 & 420 & $73-75$ & 42 \\
\hline
\end{tabular}


(DuPont Co.), and aluminum foil.(7,8) In this study, a total of twelve different sensing probes were prepared: three different shapes of sensing probes with three different reflectors, and three different shapes of sensing probes without reflectors. To measure gamma radiation, a cesium-137 source (1 $\mu \mathrm{Ci}$ ) in a disk shape, $26 \mathrm{~mm}$ in diameter, was attached to the end of the probes.

In this study, a plastic optical fiber (NY02, Edmund Optics) with a diameter of $3 \mathrm{~mm}$ and a length of $10 \mathrm{~m}$ was selected to transmit light generated in the sensing probe to the signal acquisition device. The plastic optical fiber consists of a core made of polymethylmethacrylate (PMMA) and a cladding made of fluorinated polymethyl methacrylate (F-PMMA). The properties of the plastic optical fiber chosen in this study are listed in Table 2.

The signal acquisition device used in this paper consisted of a photomultiplier tube (PMT, H5211 A, Hamamatsu), a pre-amplifier (2006, Canberra), an amplifier (AMP, 2012, Canberra), and a power supply (C3830, Hamamatsu). The multi-port II of Canberra was used as the multi-channel analyzer. Figure 2 shows the experimental setup. The bending angle of three FOSs was set to be the same so that each optical fiber would have the same bending loss.

Table 2

Properties of the plastic optical fiber.

\begin{tabular}{lc}
\hline Outer diameter $(\mathrm{mm})$ & 3.0 \\
Core diameter $(\mathrm{mm})$ & 2.95 \\
Index of refraction $n_{\mathrm{d}}$-core & 1.49 \\
Index of refraction $n_{\mathrm{d}}$-cladding & 1.402 \\
Numerical aperture, NA & 0.50 \\
Attenuation $(\mathrm{dB} / \mathrm{km})$ & $150-300($ at $650 \mathrm{~nm})$ \\
Operating temperature $\left({ }^{\circ} \mathrm{C}\right)$ & -55 to +70 \\
Minimum bend radius $(\mathrm{mm})$ & 75 \\
\hline
\end{tabular}
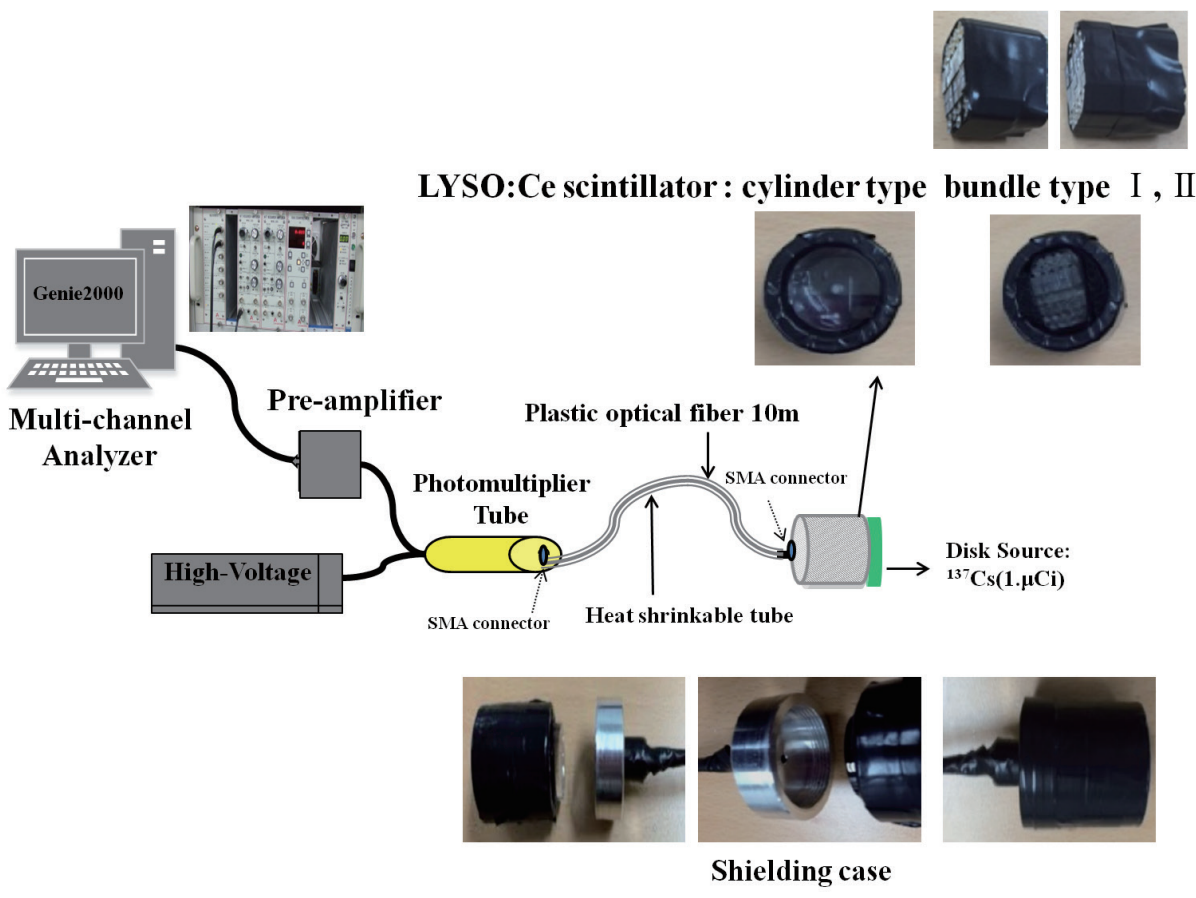

Fig. 2. (Color online) Experimental setup. 


\section{Results and Discussion}

Figures 3 to 5 show the measurements made with the twelve different FOSs developed in this study. Each figure also shows the differences in measurements according to the different shapes and reflectors of the sensing probes. As shown in the figures, all the shapes of the pulse height spectra were similar, but their peak values were different. The measurements with Bundle shape II of the sensing probe were higher by about $10 \%$ than those made by the two other shapes of probe in the case of "without reflector", and were higher by 12 to $30 \%$ than those made by the two other shapes of probe in the case of "with reflector".

Figure 6 shows the total counts collected by the FOSs with different shapes and reflectors of sensing probes, including the case of "without reflector". The total counts collected by Bundle shape II of the sensing probes were higher than those collected by the two other shapes of sensing

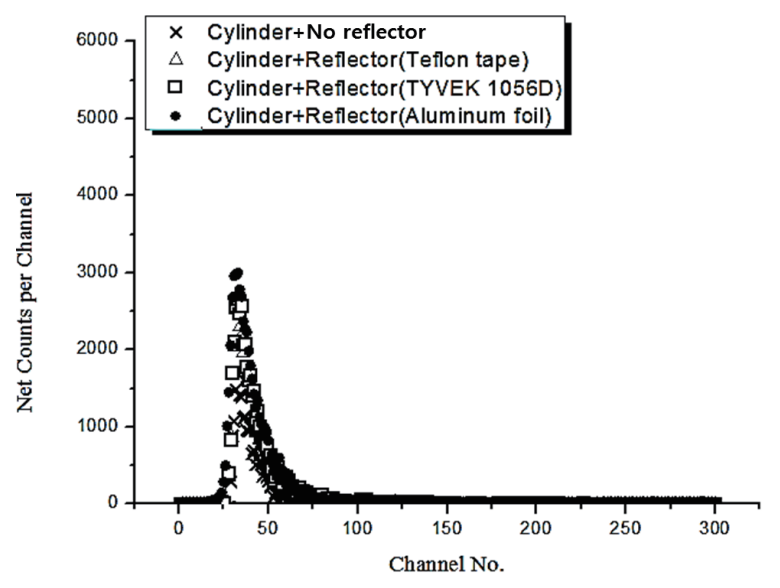

Fig. 3. The pulse height spectra of the cylindershaped sensing probes.

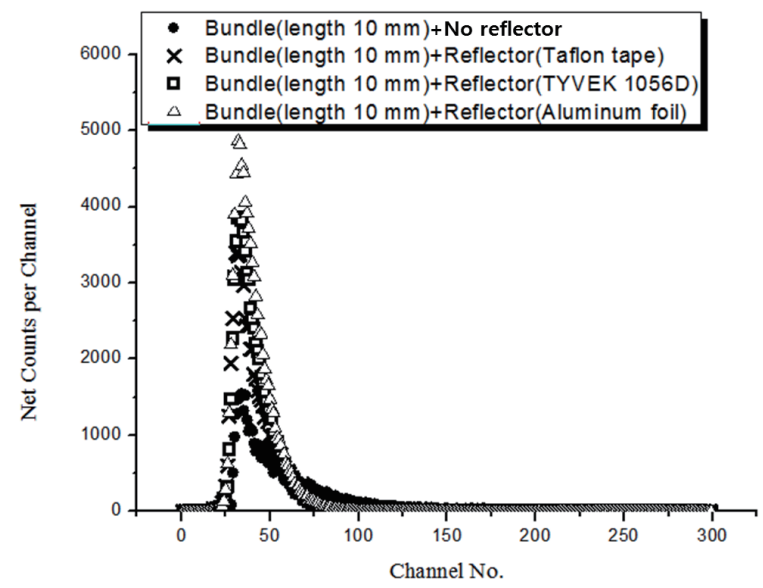

Fig. 5. The pulse height spectra of the bundleshaped sensing probes $10 \mathrm{~mm}$ in length (Bundle shape II).

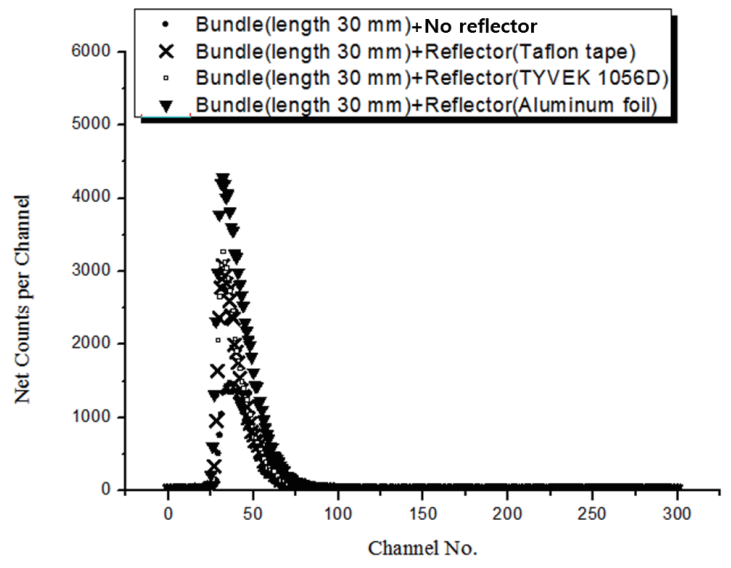

Fig. 4. The pulse height spectra of the bundleshaped sensing probes $30 \mathrm{~mm}$ in length (Bundle shape I).

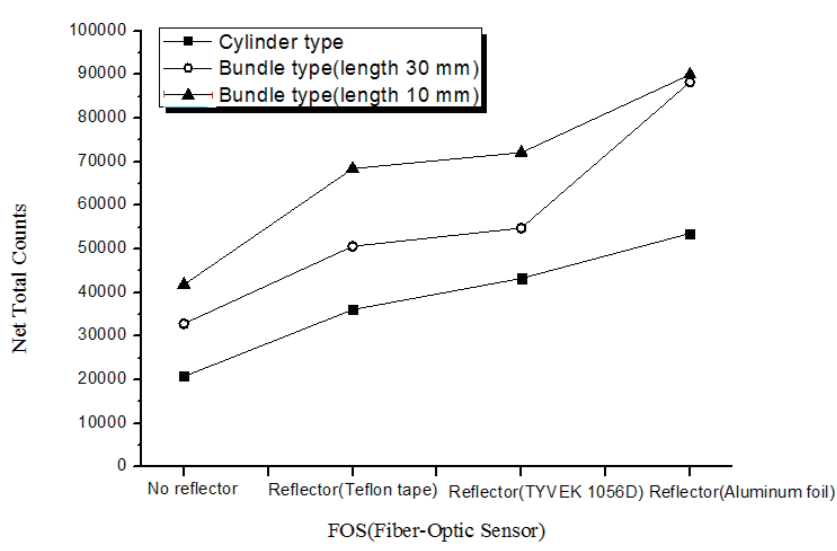

Fig. 6. Total counts by the FOSs with/without reflectors. 
probe. Comparisons between measurements showed that the total counts collected by cylindershaped sensing probes were lower by $25-50 \%$ than those collected by the two other shapes of probe. The differences in measurements between Bundle shape I and Bundle shape II were about $20 \%$, except in the case of aluminum foil reflectors. This implies that Bundle shape II was the best shape for a sensing probe, and that the aluminum foil was the best reflector in terms of FOS detection efficiency.

For remote measurement of gamma radiation, an FOS was developed in this study by combining a plastic optical fiber and an inorganic scintillator. Using the inorganic scintillator, three different shapes of sensing probe were fabricated and evaluated to identify the best shape of sensing probe in terms of its detection efficiency: a cylinder and two bundles with different heights $(30$ and $10 \mathrm{~mm}$ ). The side of each sensing probe was wrapped with one of the three different reflectors: Teflon tape, TYVEK 1056D, and aluminum foil. For the measurements of gamma radiation from a cesium-137 source in a disk shape, it was found that Bundle shape II (a bundle of 26 inorganic scintialltors, $1 \mathrm{~mm}$ in diameter and $10 \mathrm{~mm}$ in length) was the best shape for the FOS sensing probe, and that aluminum foil was the best reflector.

\section{Acknowledgements}

This work was supported by the Nuclear Safety Research Program through the Korea Foundation of Nuclear Safety (KOFONS), granted financial resource from the Nuclear Safety and Security Commission (NSSC), Republic of Korea (Research Project No. 1305017-0315-WT111).

\section{References}

1 A. F. Fernandez, B. Brichard, S. O’Keeffe, C. Fitzpatrick, E. Lewis, J. R. Vaille, L. Dusseau, D. A. Jackson, F. Ravotti, M. Glaser, and H. El-Rabii: Fusion Eng. Des. 83 (2008) 50.

2 L. T. Vo, G. D. Papworth, P. M. Delaney, D. H. Barkla, and R. G. King: J. Dermatological Sci. 23 (2000) 46.

3 B. Brichard, A. F.Fernandez, H. Ooms, P. Borgermans, and F. Berghmans: Second Eur. Workshop Opt. Fibre Sens. 5502 (2004) 184.

4 C. W. E. van Eijk: Phys. Med. Biol. 47 (2002) R85.

5 J. A. Mares, A. Beitlerova, M. Nikl, N. Solovieva, C. D’Ambrosio, K. Blazek, P. Maly, K. Nejezchleb, and F. de Notaristefani: Radiat. Meas. 38 (2004) 353.

6 S. Blahuta, A. Bessiere, D. Gourier, V. Ouspenski, and B. Viana: Opt. Mater. 35 (2013) 1865.

7 C. H. Park, J. H. Moon, and B. K. Seo: Radiat. Meas. 46 (2011) 687.

8 W. J. Yoo, S. H. Shin, D. E. Lee, K. W. Jang, S. Cho, and B. Lee: Sensors 15 (2015) 21265. 